KYUNGPOOK Math. J. 53(2013), 307-318

http://dx.doi.org/10.5666/KMJ.2013.53.3.307

\title{
Iterative Algorithm for a New System of Variational Inclu- sions with $B$-monotone Operators in Banach Spaces
}

\author{
SAng Keun LeE \\ Department of Mathematics Education, Gyeongsang National University, Jinju 660- \\ 701, South Korea \\ e-mail : sklee@gnu.kr \\ JAE UG JEONG* \\ Department of Mathematics, Dongeui University, Busan 614-714, South Korea \\ e-mail : jujeong@deu.ac.kr
}

ABSTRACT. In this paper, we introduce and study a new system of variational inclusions with $B$-monotone operators in Banach spaces. By using the proximal mapping associated with $B$ - monotone operator, we construct a new iterative algorithm for approximating the solution of this system of variational inclusions. We also prove the existence of solutions and the convergence of the sequences generated by the algorithm for this system of variational inclusions. The results presented in this paper extend and improve some known results in the literature.

\section{Introduction}

In recent years, variational inclusion theory has emerged one of the main branch of mathematical and engineering sciences. This theory provides us with a simple, natural, unified and general framework to study a wide class of unrelated problems in media, elasticity, transportation, economics, optimization, regional, physical, structural and applied science. For details see [1-9,11] and references therein.

In 2010, Luo and Huang [9] introduced and studied the following variational inclusions with $B$-monotone operators in Banach spaces:

Suppose that $E$ is a reflexive Banach space with the topological dual space $E^{*}$, $A: E \rightarrow E^{*}, N: E \times E \times E \rightarrow E^{*}, f, g: E \rightarrow E$ are single-valued mappings, $S, T, W: E \rightarrow C B(E)$ are set-valued mappings and $M: E \times E \rightarrow 2^{E^{*}}$ is a $B$ monotone mapping, where $C B(E)$ denotes the family of all nonempty closed and bounded subsets of $E$. For any given $a \in E^{*}$, find $x \in E, s \in S(x), t \in T(x)$ and

* Corresponding Author.

Received Novemver 22, 2010; accepted April 2, 2013.

2010 Mathematics Subject Classification: 49J40, 90C30.

Key words and phrases: $B$-monotone operator, Proximal mapping, Iterative algorithm, Variational inclusion, Convergence. 
$w \in W(x)$ such that

$$
a \in A(x)+M(f(x), g(x))-N(s, t, w) .
$$

Inspired and motivated by the results in Luo and Huang [9], the purpose of this paper is to introduce and study a new system of generalized variational inclusions with $B$-monotone operators in Banach spaces. By using the technique of the proximal mapping, we establish the equivalence between the generalized variational inclusions and the proximal mapping equations in reflexive Banach spaces. We use this equivalence and Nadler's theorem [10] to construct a new iterative algorithm for solving this system of generalized variational inclusions. And we prove the existence of solutions for this system of generalized variational inclusions. We also prove the convergence of an iterative algorithm approximating the solution for this system of generalized variational inclusions in reflexive Banach spaces. The results in this paper unify, extend and improve some results from $[6,8,9,11]$.

\section{Preliminaries}

Throughout this paper we assume that $E$ is a real Banach space with dual space $E^{*},\langle\cdot, \cdot\rangle$ is the dual pair between $E$ and $E^{*}, 2^{E^{*}}$ denotes the family of all

the nonempty subsets of $E^{*}$ and $C B(E)$ is the family of all nonempty closed and bounded subsets of $E$.

Definition 2.1. Let $A: E \rightarrow E^{*}$ be a single-valued mapping. $A$ is said to be

(i) monotone if

$$
\langle A(x)-A(y), x-y\rangle \geq 0, \quad \forall x, y \in E,
$$

(ii) strictly monotone if

$$
\langle A(x)-A(y), x-y\rangle \geq 0, \quad \forall x, y \in E,
$$

and equality holds if and only if $x=y$.

Definition 2.2. Let $T: E \rightarrow 2^{E^{*}}$ be a multi-valued mapping. $T$ is said to be

(i) monotone if

$$
\langle u-v, x-y\rangle \geq 0, \quad \forall x, y \in E, u \in T x, v \in T y
$$

(ii) strictly monotone if

$$
\langle u-v, x-y\rangle \geq 0, \quad \forall x, y \in E, u \in T x, v \in T y,
$$

and equality holds if and only if $x=y$;

(iii) $r$-strongly monotone if there exists a constant $r>0$ such that

$$
\langle u-v, x-y\rangle \geq r\|x-y\|^{2}, \quad \forall x, y \in E, u \in T x, v \in T y
$$


(iv) $s$-relaxed monotone if there exists a constant $s>0$ such that

$$
\langle u-v, x-y\rangle \geq-s\|x-y\|^{2}, \quad \forall x, y \in E, u \in T x, v \in T y .
$$

Definition 2.3. Let $M: E \times E \rightarrow 2^{E^{*}}$ be a multi-valued mapping and $f, g: E \rightarrow E$ be single-valued mappings.

(i) $M(f, \cdot)$ is said to be $\alpha$-strongly monotone with respect to $f$ if there exists a constant $\alpha>0$ such that

$\langle u-v, x-y\rangle \geq \alpha\|x-y\|^{2}, \quad \forall x, y, \omega \in E, u \in M(f(x), \omega), v \in M(f(y), \omega)$,

(ii) $M(\cdot, g)$ is said to be $\beta$-relaxed monotone with respect to $g$ if there exists a constant $\beta>0$ such that

$$
\langle u-v, x-y\rangle \geq-\beta\|x-y\|^{2}, \quad \forall x, y, \omega \in E, u \in M(\omega, g(x)), v \in M(\omega, g(y)),
$$

(iii) $M(\cdot, \cdot)$ is said to be $(\alpha, \beta)$-symmetric monotone with respect to $f$ and $g$ if $M(f, \cdot)$ is $\alpha$-strongly monotone with respect to $f$ and $M(\cdot, g)$ is $\beta$-relaxed monotone with respect to $g$ with $\alpha \geq \beta$ and $\alpha=\beta$ if and only if $x=y$.

Definition 2.4([9]). Let $E$ be a Banach space with the dual space $E^{*}$. Let $f, g: E \rightarrow E, B: E \rightarrow E^{*}$ be single-valued mappings and $M: E \times E \rightarrow 2^{E^{*}}$ be a multi-valued mapping. Then $M$ is said to be $B$-monotone if $M$ is $(\alpha, \beta)$-symmetric monotone with respect to $f$ and $g$ and $(B+\rho M(f, g))(E)=E^{*}$ for every $\rho>0$.

Definition 2.5. Let $E$ be a reflexive Banach space with the dual space $E^{*}$. Let $f, g: E \rightarrow E$ be single-valued mappings, $B: E \rightarrow E^{*}$ be a strictly monotone mapping and $M: E \times E \rightarrow 2^{E^{*}}$ be a $B$-monotone mapping and $\rho>0$ be a constant. Then a proximal mapping $R_{M(\cdot, \cdot), \rho}^{B}: E^{*} \rightarrow E$ is defined by

$$
R_{M(\cdot, \cdot), \rho}^{B}\left(x^{*}\right)=(B+\rho M(f, g))^{-1}\left(x^{*}\right), \quad \forall x^{*} \in E^{*} .
$$

Remark 2.1. If $M(f, g)=M$ and $M$ is a general $H$-monotone, then the proximal mapping $R_{M(\cdot,), \rho}^{B}$ reduces to the proximal mapping $R_{M}^{H}$ considered in [6,11].

Definition 2.6. A multi-valued mapping $T: E \rightarrow C B(E)$ is said to be $D$-Lipschitz continuous if there exists a constant $h>0$ such that

$$
D(T(x), T(y)) \leq h\|x-y\|, \quad \forall x, y \in E,
$$

where $D(\cdot, \cdot)$ denotes the Hausdorff metric on $C B(E)$.

Lemma 2.1([9]). Let $E$ be a reflexive Banach space with the dual space $E^{*}$. Let 
$f, g: E \rightarrow E$ be single-valued mappings, $B: E \rightarrow E^{*}$ be a strictly monotone mapping and $M: E \times E \rightarrow 2^{E^{*}}$ be a $B$-monotone mapping. Then the proximal mapping $R_{M(\cdot, \cdot), \rho}^{B}: E^{*} \rightarrow E$ is Lipschitz continuous with constant $\frac{1}{\rho(\alpha-\beta)}$, i.e.,

$$
\left\|R_{M(\cdot, \cdot), \rho}^{B}\left(x^{*}\right)-R_{M(\cdot, \cdot), \rho}^{B}\left(y^{*}\right)\right\| \leq \frac{1}{\rho(\alpha-\beta)}\left\|x^{*}-y^{*}\right\|, \quad \forall x^{*}, y^{*} \in E^{*} .
$$

\section{A System of Generalized Variational Inclusions with $B$-monotone Mappings and Iterative Algorithm}

In this section, we will introduce a new system of generalized variational inclusions with $B$-monotone and construct a new iterative algorithm for this system.

Let $E$ be a reflexive Banach space with the dual space $E^{*}, A, B: E \rightarrow E^{*}$, $N: E \times E \times E \rightarrow E^{*}, f, g: E \rightarrow E$ be a single-valued mappings, $M_{1}, M_{2}: E \times E \rightarrow$ $2^{E^{*}}$ be $B$-monotone mappings, $S, T, W: E \rightarrow C B(E)$ be set-valued mappings. We consider the following problem: for any given $a_{1}, a_{2} \in E$ find $x, y \in E, s \in S(x)$, $\bar{s} \in S(y), t \in T(x), \bar{t} \in T(y), w \in W(x)$ and $\bar{w} \in W(y)$ such that

$$
\begin{aligned}
& \rho a_{1} \in B(x)-B(y)+\rho\left[A(x)+M_{1}(f(x), g(x))-N(s, t, w)\right], \\
& \lambda a_{2} \in B(y)-B(x)+\lambda\left[A(x)+M_{2}(f(y), g(y))-N(\bar{s}, \bar{t}, \bar{w})\right],
\end{aligned}
$$

where $\rho, \lambda>0$ are constants.

Problem (3.1) is called a system of generalized variational inclusions with $B$ monotone mappings.

Below are some special cases of problem (3.1):

(I) If $x=y, \rho=1$ and $\lambda=0$, then the problem (3.1) reduces to the variational inclusion problem with $B$-monotone operators (1.1) introduced and studied by Luo and Huang [9].

(II) If $x=y, \rho=1, \lambda=0$ and $g=I$ is an identity mapping on $E$, then the problem (3.1) reduces to the following problem: for any given $a_{1} \in E^{*}$, find $x \in E$, $s \in S(x), t \in T(x)$ and $w \in W(x)$ such that

$$
a_{1} \in A(x)+M(f(x), x)-N(s, t, w) .
$$

Problem (3.2) was introduced and studied by Ding and Feng [6].

(III) If $x=y, \rho=1, \lambda=0, a_{1}=0, N=0$ and $M(f(x), g(x))=M(f(x))$, then the problem (4.1) reduces to the following problem: find $x \in E$ such that

$$
0 \in A(x)+M(f(x)) .
$$

Problem (3.3) was introduced and studied by Xia and Huang [11]. 
(IV) If $E$ is a Hilbert space, $x=y, \rho=1, \lambda=0, a_{1}=0, N=0$ and $M(f(x), g(x))=M(x)$, then the problem (4.1) reduces to the following problem: find $x \in E$ such that

$$
0 \in A(x)+M(x) .
$$

Problem (3.4) was introduced and studied by Feng and Huang [8].

Theorem 3.1. Let $A: E \rightarrow E^{*}, N: E \times E \times E \rightarrow E^{*}, f, g: E \rightarrow E$ be single-valued mappings, $S, T, W: E \rightarrow C B(E)$ be multi-valued mappings. Let $B: E \rightarrow E^{*}$ be a strictly monotone mapping and $M_{1}, M_{2}: E \times E \rightarrow 2^{E^{*}}$ be $B$-monotone mappings. Then $(x, y, s, \bar{s}, t, \bar{t}, w, \bar{w})$ with $x, y \in E, s \in S(x), \bar{s} \in S(y), t \in T(x), \bar{t} \in T(y)$, $w \in W(x)$ and $\bar{w} \in W(y)$ is a solution of problem (3.1) if and only if

$$
\begin{aligned}
& x=R_{M_{1}(\cdot, \cdot), \rho}^{B}\left[\rho a_{1}+B(y)-\rho A(x)+\rho N(s, t, w)\right], \\
& y=R_{M_{2}(\cdot, \cdot), \lambda}^{B}\left[\lambda a_{2}+B(x)-\lambda A(x)+\lambda N(\bar{s}, \bar{t}, \bar{w})\right] .
\end{aligned}
$$

Proof. The fact directly follows from Definition 2.5 and some arguments.

Remark 3.1. (i) If $x=y, \rho=1$ and $\lambda=0$, then Theorem 3.1 reduces to Theorem 4.1 in [9].

(ii) If $x=y, \rho=1, \lambda=0, g=I$ is an identity mapping on $E$ and $M$ is a general $H$-monotone mapping in the first argument, then Theorem 3.1 reduces to Theorem 3.1 in [6]

(iii) If $x=y, \rho=1, \lambda=0, a_{1}=0, N=0, M(f(x), g(x))=M(f(x))$ and $M$ is a general $H$-monotone mapping, then Theorem 3.1 reduces to Theorem 3.3 in [11].

For any given $x_{0}, y_{0} \in E$, take $s_{0} \in S\left(x_{0}\right), \bar{s}_{0} \in S\left(y_{0}\right), t_{0} \in T\left(x_{0}\right), \bar{t}_{0} \in T\left(y_{0}\right)$, $w_{0} \in W\left(x_{0}\right)$ and $\bar{w}_{0} \in W\left(y_{0}\right)$. It follows from Theorem 3.1 that there exist $x_{1}, y_{1} \in$ such that

$$
\begin{aligned}
& x_{1}=R_{M_{1}(\cdot, \cdot), \rho}^{B}\left[\rho a_{1}+B\left(y_{0}\right)-\rho A\left(x_{0}\right)+\rho N\left(s_{0}, t_{0}, w_{0}\right)\right], \\
& y_{1}=R_{M_{2}(\cdot, \cdot), \lambda}^{B}\left[\lambda a_{2}+B\left(x_{0}\right)-\lambda A\left(x_{0}\right)+\lambda N\left(\bar{s}_{0}, \bar{t}_{0}, \bar{w}_{0}\right)\right] .
\end{aligned}
$$

Since $s_{0} \in S\left(x_{0}\right), \bar{s} \in S\left(y_{0}\right), t_{0} \in T\left(x_{0}\right), \bar{t}_{0} \in T\left(y_{0}\right), w_{0} \in W\left(x_{0}\right)$ and $\bar{w}_{0} \in W\left(y_{0}\right)$, by Nadler's theorem [10], there exist $s_{1} \in S\left(x_{1}\right), \bar{s}_{1} \in S\left(x_{1}\right), t_{1} \in T\left(x_{1}\right), \bar{t}_{1} \in T\left(y_{1}\right)$, $w_{1} \in W\left(x_{1}\right)$ and $\bar{w}_{1} \in W\left(y_{1}\right)$ such that

$$
\begin{aligned}
& \| s_{1}-s_{0} \mid \leq(1+1) D\left(S\left(x_{1}\right), S\left(x_{0}\right)\right), \\
& \left\|\bar{s}_{1}-\bar{s}_{0}\right\| \leq(1+1) D\left(S\left(y_{1}\right), S\left(y_{0}\right)\right), \\
& \left\|t_{1}-t_{0}\right\| \leq(1+1) D\left(T\left(x_{1}\right), T\left(x_{0}\right)\right),
\end{aligned}
$$




$$
\begin{gathered}
\left\|\bar{t}_{1}-\bar{t}_{0}\right\| \leq(1+1) D\left(T\left(y_{1}\right), T\left(y_{0}\right)\right), \\
\left\|w_{1}-w_{0}\right\| \leq(1+1) D\left(W\left(x_{1}\right), W\left(x_{0}\right)\right), \\
\left\|\bar{w}_{1}-\bar{w}_{0}\right\| \leq(1+1) D\left(W\left(y_{1}\right), W\left(y_{0}\right)\right) .
\end{gathered}
$$

Hence we can construct an iterative algorithm for solving problem (3.1) as follows: Algorithm 3.1. For any given $x_{0}, y_{0} \in E, s_{0} \in S\left(x_{0}\right), \bar{s}_{0} \in S\left(y_{0}\right), t_{0} \in T\left(x_{0}\right)$, $\bar{t}_{0} \in T\left(y_{0}\right), w_{0} \in W\left(x_{0}\right), \bar{w} \in W\left(y_{0}\right)$, we can obtain the sequence $\left\{x_{n}\right\},\left\{y_{n}\right\},\left\{s_{n}\right\}$, $\left\{\bar{s}_{n}\right\},\left\{t_{n}\right\},\left\{\bar{t}_{n}\right\},\left\{w_{n}\right\},\left\{\bar{w}_{n}\right\}$ such that

$$
\begin{gathered}
x_{n+1}=R_{M_{1}(\cdot, \cdot), \rho}^{B}\left[\rho a_{1}+B\left(y_{n}\right)-\rho A\left(x_{n}\right)+\rho N\left(s_{n}, t_{n}, w_{n}\right)\right], \\
y_{n+1}=R_{M_{2}(\cdot, \cdot), \lambda}^{B}\left[\lambda a_{2}+B\left(x_{n}\right)-\lambda A\left(x_{n}\right)+\lambda N\left(\bar{s}_{n}, \bar{t}_{n}, \bar{w}_{n}\right)\right], \\
s_{n} \in S\left(x_{n}\right), \quad\left\|s_{n+1}-s_{n}\right\| \leq\left(1+\frac{1}{n+1}\right) D\left(S\left(x_{n+1}\right), S\left(x_{n}\right)\right), \\
\bar{s}_{n} \in S\left(y_{n}\right), \quad\left\|\bar{s}_{n+1}-\bar{s}_{n}\right\| \leq\left(1+\frac{1}{n+1}\right) D\left(S\left(y_{n+1}\right), S\left(y_{n}\right)\right), \\
t_{n} \in T\left(x_{n}\right), \quad\left\|t_{n+1}-t_{n}\right\| \leq\left(1+\frac{1}{n+1}\right) D\left(T\left(x_{n+1}\right), T\left(x_{n}\right)\right), \\
\bar{t}_{n} \in T\left(y_{n}\right), \quad\left\|\bar{t}_{n+1}-\bar{t}_{n}\right\| \leq\left(1+\frac{1}{n+1}\right) D\left(T\left(y_{n+1}\right), T\left(y_{n}\right)\right), \\
w_{n} \in W\left(x_{n}\right), \quad\left\|w_{n+1}-w_{n}\right\| \leq\left(1+\frac{1}{n+1}\right) D\left(W\left(x_{n+1}\right), W\left(x_{n}\right)\right), \\
\bar{w}_{n} \in W\left(y_{n}\right), \quad\left\|\bar{w}_{n+1}-\bar{w}_{n}\right\| \leq\left(1+\frac{1}{n+1}\right) D\left(W\left(y_{n+1}\right), W\left(y_{n}\right)\right) .
\end{gathered}
$$

\section{Convergence of an Iterative Algorithm}

In this section, we show the existence of solutions for problem (3.1) and the convergence of the iterative sequences generated by Algorithm 3.1.

Theorem 4.1. Let $E$ be a reflexive Banach space with the dual space $E^{*}$. Let $f, g: E \rightarrow E$ be single-valued mappings, $A: E \rightarrow E^{*}$ be a $\tau$-Lipschitz continuous mapping, $B: E \rightarrow E^{*}$ be a strictly monotone and $\delta$-Lipschitz continuous mapping 
and $M_{1}, M_{2}: E \times E \rightarrow 2^{E^{*}}$ be B-monotone mappings. Let $S, T, W: E \rightarrow C B(E)$ be D-Lipschitz continuous with constants $k_{S}>0, k_{T}>0, k_{W}>0$, respectively and $N: E \times E \times E \rightarrow E^{*}$ be $\xi_{1}$-Lipschitz continuous in the first argument, $\xi_{2}$-Lipschitz continuous in the second argument, $\xi_{3}$-Lipschitz continuous in the third argument. If there exist constants $\rho, \lambda>0$ such that

$$
\begin{gathered}
\frac{1}{\alpha-\beta}\left\{\tau+\xi_{1} k_{S}+\xi_{2} k_{T}+\xi_{3} k_{W}+\frac{1}{\lambda}(\delta+\lambda \tau)\right\}<1, \\
\frac{1}{\alpha-\beta}\left\{\frac{\delta}{\rho}+\xi_{1} k_{S}+\xi_{2} k_{T}+\xi_{3} k_{W}\right\}<1,
\end{gathered}
$$

then problem (3.1) has a solution $(x, y, s, \bar{s}, t, \bar{t}, w, \bar{w})$ and sequences $\left\{x_{n}\right\},\left\{y_{n}\right\}$, $\left\{s_{n}\right\},\left\{\bar{s}_{n}\right\},\left\{t_{n}\right\},\left\{\bar{t}_{n}\right\},\left\{w_{n}\right\},\left\{\bar{w}_{n}\right\}$ converge to $x, y, s, \bar{s}, t, \bar{t}, w, \bar{w}$, respectively, where $\left\{x_{n}\right\},\left\{y_{n}\right\},\left\{s_{n}\right\},\left\{\bar{s}_{n}\right\},\left\{t_{n}\right\},\left\{\bar{t}_{n}\right\},\left\{w_{n}\right\},\left\{\bar{w}_{n}\right\}$ are the sequences generated by Algorithm 3.1.

Proof. For $n=1,2, \cdots$, let

$$
\begin{aligned}
& \Omega_{n}=\rho a_{1}+B\left(y_{n}\right)-\rho A\left(x_{n}\right)+\rho N\left(s_{n}, t_{n}, w_{n}\right), \\
& \Delta_{n}=\lambda a_{2}+B\left(x_{n}\right)-\lambda A\left(x_{n}\right)+\lambda N\left(\bar{s}_{n}, \bar{t}_{n}, \bar{w}_{n}\right) .
\end{aligned}
$$

By (3.3) and Lemma 2.2, we have

$$
\begin{aligned}
\left\|x_{n+1}-x_{n}\right\| & =\left\|R_{M(\cdot, \cdot), \rho}^{B}\left(\Omega_{n}\right)-R_{M(\cdot, \cdot), \rho}^{B}\left(\Omega_{n-1}\right)\right\| \\
& \leq \frac{1}{\rho(\alpha-\beta)}\left\|\Omega_{n}-\Omega_{n-1}\right\|, \\
\left\|y_{n+1}-y_{n}\right\| & =\left\|R_{M(\cdot, \cdot), \lambda}^{B}\left(\Delta_{n}\right)-R_{M(\cdot, \cdot), \lambda}^{B}\left(\Delta_{n-1}\right)\right\| \\
& \leq \frac{1}{\lambda(\alpha-\beta)}\left\|\Delta_{n}-\Delta_{n-1}\right\| .
\end{aligned}
$$

And

$$
\begin{aligned}
\left\|\Omega_{n}-\Omega_{n-1}\right\| \leq & \left\|B\left(y_{n}\right)-B\left(y_{n-1}\right)\right\|+\rho \| A\left(x_{n}-A\left(x_{n-1}\right) \|\right. \\
& +\rho\left\|N\left(s_{n}, t_{n}, w_{n}\right)-N\left(s_{n-1}, t_{n-1}, w_{n-1}\right)\right\|, \\
\left\|\Delta_{n}-\Delta_{n-1}\right\| \leq & \left\|B\left(x_{n}\right)-B\left(x_{n-1}\right)\right\|+\lambda\left\|A\left(x_{n}\right)-A\left(x_{n-1}\right)\right\| \\
& +\lambda\left\|N\left(\bar{s}_{n}, \bar{t}_{n}, \bar{w}_{n}\right)-N\left(\bar{s}_{n-1}, \bar{t}_{n-1}, \bar{w}_{n-1}\right)\right\| .
\end{aligned}
$$

Using the Lipschitz continuities of $A, B, N, S, T$ and $W$ we obtain

$$
\left\|B\left(x_{n}\right)-B\left(x_{n-1}\right)\right\| \leq \delta\left\|x_{n}-x_{n-1}\right\|,
$$




$$
\begin{aligned}
& \left\|B\left(y_{n}\right)-B\left(y_{n_{1}}\right)\right\| \leq \delta\left\|y_{n}-y_{n-1}\right\|, \\
& \left\|A\left(x_{n}\right)-A\left(x_{n-1}\right)\right\| \leq \tau\left\|x_{n}-x_{n-1}\right\|, \\
& \left\|s_{n}-s_{n-1}\right\| \leq\left(1+\frac{1}{n}\right) D\left(S\left(x_{n}\right), S\left(x_{n-1}\right)\right) \\
& \leq\left(1+\frac{1}{n}\right) k_{S}\left\|x_{n}-x_{n-1}\right\|, \\
& \left\|\bar{s}_{n}-\bar{s}_{n-1}\right\| \leq\left(1+\frac{1}{n}\right) D\left(S\left(y_{n}\right), S\left(y_{n-1}\right)\right) \\
& \leq\left(1+\frac{1}{n}\right) k_{S}\left\|y_{n}-y_{n-1}\right\|, \\
& \left\|t_{n}-t_{n-1}\right\| \leq\left(1+\frac{1}{n}\right) D\left(T\left(x_{n}\right), T\left(x_{n-1}\right)\right) \\
& \leq\left(1+\frac{1}{n}\right) k_{T}\left\|x_{n}-x_{n-1}\right\|, \\
& \left\|\bar{t}_{n}-\bar{t}_{n-1}\right\| \leq\left(1+\frac{1}{n}\right) D\left(T\left(y_{n}\right), T\left(y_{n-1}\right)\right) \\
& \leq\left(1+\frac{1}{n}\right) k_{T}\left\|y_{n}-y_{n-1}\right\|, \\
& \left\|w_{n}-w_{n-1}\right\| \leq\left(1+\frac{1}{n}\right) D\left(W\left(x_{n}\right), W\left(x_{n-1}\right)\right) \\
& \leq\left(1+\frac{1}{n}\right) k_{W}\left\|x_{n}-x_{n-1}\right\|, \\
& \left\|\bar{w}_{n}-\bar{w}_{n-1}\right\| \leq\left(1+\frac{1}{n}\right) D\left(W\left(y_{n}\right), W\left(y_{n-1}\right)\right) \\
& \leq\left(1+\frac{1}{n}\right) k_{W}\left\|y_{n}-y_{n-1}\right\|, \\
& \left\|N\left(s_{n}, t_{n}, w_{n}\right)-N\left(s_{n-1}, t_{n-1}, w_{n-1}\right)\right\| \\
& \leq\left\|N\left(s_{n}, t_{n}, w_{n}\right)-N\left(s_{n-1}, t_{n}, w_{n}\right)\right\| \\
& \left.+\| N\left(s_{n-1}, t_{n}, w_{n}\right)\right)-N\left(s_{n-1}, t_{n-1}, w_{n}\right) \| \\
& +\left\|N\left(s_{n-1}, t_{n-1}, w_{n}\right)-N\left(s_{n-1}, t_{n-1}, w_{n-1}\right)\right\| \\
& \leq \xi_{1}\left\|s_{n}-s_{n-1}\right\|+\xi_{2}\left\|t_{n}-t_{n-1}\right\|+\xi_{3}\left\|w_{n}-w_{n-1}\right\| \\
& \leq\left(1+\frac{1}{n}\right)\left(\xi_{1} k_{S}+\xi_{2} k_{T}+\xi_{3} k_{W}\right)\left\|x_{n}-x_{n-1}\right\|,
\end{aligned}
$$




$$
\begin{aligned}
\| & N\left(\bar{s}_{n}, \bar{t}_{n}, \bar{w}_{n}\right)-N\left(\bar{s}_{n-1}, \bar{t}_{n-1}, \bar{w}_{n-1}\right) \| \\
\leq & \left\|N\left(\bar{s}_{n}, \bar{t}_{n}, \bar{w}_{n}\right)-N\left(\bar{s}_{n-1}, \bar{t}_{n}, \bar{w}_{n}\right)\right\| \\
& +\left\|N\left(\bar{s}_{n-1}, \bar{t}_{n}, \bar{w}_{n}\right)-N\left(\bar{s}_{n-1}, \bar{t}_{n-1}, \bar{w}_{n}\right)\right\| \\
& +\left\|N\left(\bar{s}_{n-1}, \bar{t}_{n-1}, \bar{w}_{n}\right)-N\left(\bar{s}_{n-1}, \bar{t}_{n-1}, \bar{w}_{n-1}\right)\right\| \\
\leq & \xi_{1}\left\|\bar{s}_{n}-\bar{s}_{n-1}\right\|+\xi_{2}\left\|\bar{t}_{n}-\bar{t}_{n-1}\right\|+\xi_{3}\left\|\bar{w}_{n}-\bar{w}_{n-1}\right\| \\
\leq & \left(1+\frac{1}{n}\right)\left(\xi_{1} k_{S}+\xi_{2} k_{T}+\xi_{3} k_{W}\right)\left\|y_{n}-y_{n-1}\right\| .
\end{aligned}
$$

It follows from (4.3)-(4.18) that

$$
\begin{aligned}
& \left\|x_{n+1}-x_{n}\right\| \\
& \leq \frac{1}{\rho(\alpha-\beta)}\left[\left\|B\left(y_{n}\right)-B\left(y_{n-1}\right)\right\|+\rho\left\|A\left(x_{n}\right)-A\left(x_{n-1}\right)\right\|\right. \\
& \left.+\rho\left\|N\left(s_{n}, t_{n}, w_{n}\right)-N\left(s_{n-1}, t_{n-1}, w_{n-1}\right)\right\|\right] \\
& \leq \frac{1}{\rho(\alpha-\beta)}\left[\delta\left\|y_{n}-y_{n-1}\right\|+\rho \tau\left\|x_{n}-x_{n-1}\right\|\right. \\
& \left.+\rho\left(1+\frac{1}{n}\right)\left(\xi_{1} k_{S}+\xi_{2} k_{T}+\xi_{3} k_{W}\right)\left\|x_{n}-x_{n-1}\right\|\right], \\
& \left\|y_{n+1}-y_{n}\right\| \\
& \leq \frac{1}{\lambda(\alpha-\beta)}\left[\| B\left(x_{n}-B\left(x_{n-1}\right)\|+\lambda\| A\left(x_{n}\right)-A\left(x_{n-1}\right) \|\right.\right. \\
& \left.+\lambda\left\|N\left(\bar{s}_{n}, \bar{t}_{n}, \bar{w}_{n}\right)-N\left(\bar{s}_{n-1}, \bar{t}_{n-1}, \bar{w}_{n-1}\right)\right\|\right] \\
& \leq \frac{1}{\lambda(\alpha-\beta)}\left[\delta\left\|x_{n}-x_{n-1}\right\|+\lambda \tau\left\|x_{n}-x_{n-1}\right\|\right. \\
& \left.+\lambda\left(1+\frac{1}{n}\right)\left(\xi_{1} k_{S}+\xi_{2} k_{T}+\xi_{3} k_{W}\right)\left\|y_{n}-y_{n-1}\right\|\right] .
\end{aligned}
$$

Thus

$$
\begin{aligned}
& \left\|x_{n+1}-x_{n}\right\|+\left\|y_{n+1}-y_{n}\right\| \\
& \leq \frac{1}{\alpha-\beta}\left[\tau+\left(1+\frac{1}{n}\right)\left(\xi_{1} k_{S}+\xi_{2} k_{T}+\xi_{3} k_{W}\right)+\frac{1}{\lambda}(\delta+\lambda \tau)\right]\left\|x_{n}-x_{n-1}\right\| \\
& \quad+\frac{1}{\alpha-\beta}\left[\frac{\delta}{\rho}+\left(1+\frac{1}{n}\right)\left(\xi_{1} k_{S}+\xi_{2} k_{T}+\xi_{3} k_{W}\right)\right]\left\|y_{n}-y_{n-1}\right\| \\
& \leq \theta_{n}\left[\left\|x_{n}-x_{n-1}\right\|+\left\|y_{n}-y_{n-1}\right\|\right],
\end{aligned}
$$

where

$$
\begin{gathered}
\theta_{n}=\max \left\{\frac{1}{\alpha-\beta}\left[\tau+\left(1+\frac{1}{n}\right)\left(\xi_{1} k_{S}+\xi_{2} k_{T}+\xi_{3} k_{W}\right)+\frac{1}{\lambda}(\delta+\lambda \tau)\right],\right. \\
\left.\frac{1}{\alpha-\beta}\left[\frac{\delta}{\rho}+\left(1+\frac{1}{n}\right)\left(\xi_{1} k_{S}+\xi_{2} k_{T}+\xi_{3} k_{W}\right)\right]\right\} .
\end{gathered}
$$


Let

$$
\begin{gathered}
\theta=\max \left\{\frac{1}{\alpha-\beta}\left[\tau+\xi_{1} k_{S}+\xi_{2} k_{T}+\xi_{3} k_{W}+\frac{1}{\lambda}(\delta+\lambda \tau)\right],\right. \\
\left.\frac{1}{\alpha-\beta}\left[\frac{\delta}{\rho}+\xi_{1} k_{S}+\xi_{2} k_{T}+\xi_{3} k_{W}\right]\right\} .
\end{gathered}
$$

Then $\theta_{n} \rightarrow \theta$ as $n \rightarrow \infty$. By (4.1)-(4.2), we know that $0<\theta<1$ and hence there exists an $n_{0}>0$ and $\theta_{0} \in(0,1)$ such that $\theta_{n} \leq \theta_{0}$ for all $n \geq n_{0}$. Therefore by (4.21), we have

$$
\begin{aligned}
& \left\|x_{n+1}-x_{n}\right\|+\left\|y_{n+1}-y_{n}\right\| \\
& \leq \theta_{0}\left[\left\|x_{n}-x_{n-1}\right\|+\left\|y_{n}-y_{n-1}\right\|\right] \\
& \leq \theta_{0}^{n-n_{0}}\left[\left\|x_{n_{0}+1}-x_{n_{0}}\right\|+\left\|y_{n_{0}+1}-y_{n_{0}}\right\|\right], \quad \forall n \geq n_{0} .
\end{aligned}
$$

Hence for any $m \geq n \geq n_{0}$, it follows that

$$
\begin{aligned}
\left\|x_{m}-x_{n}\right\| & \leq \sum_{i=n}^{m-1}\left[\left\|x_{i+1}-x_{i}\right\|+\left\|y_{i+1}-y_{i}\right\|\right] \\
& \leq \sum_{i=n}^{m-1} \theta_{0}^{i-n_{0}}\left[\left\|x_{n_{0}+1}-x_{n_{0}}\right\|+\| y_{n_{0}+1}-y_{n_{0}}\right] \| .
\end{aligned}
$$

Since $0<\theta_{0}<1$, it follows from (4.22) that

$$
\left\|x_{m}-x_{n}\right\| \rightarrow 0 \quad \text { as } n \rightarrow \infty
$$

and hence $\left\{x_{n}\right\}$ is a Cauchy sequence in $E$. By the same argument, we also have that $\left\{y_{n}\right\}$ is a Cauchy sequence. Thus, there exist $x, y \in E$ such that $x_{n} \rightarrow x$, $y_{n} \rightarrow y$ as $n \rightarrow \infty$.

Now we prove that $s_{n} \rightarrow s \in S(x), \bar{s}_{n} \rightarrow \bar{s} \in S(y), t_{n} \rightarrow t \in T(x), \bar{t}_{n} \rightarrow \bar{t} \in$ $T(y), w_{n} \rightarrow w \in W(x)$ and $\bar{w}_{n} \rightarrow \bar{w} \in W(y)$ as $n \rightarrow \infty$.

In fact, it follows from (4.10)-(4.15) that $\left\{s_{n}\right\},\left\{\bar{s}_{n}\right\},\left\{t_{n}\right\},\left\{\bar{t}_{n}\right\},\left\{w_{n}\right\},\left\{\bar{w}_{n}\right\}$ are also Cauchy sequences. Therefore there exist $s, \bar{s}, t, \bar{t}, w, \bar{w} \in E$ such that $s_{n} \rightarrow s$, $\bar{s}_{n} \rightarrow \bar{s}, t_{n} \rightarrow t, \bar{t}_{n} \rightarrow \bar{t}, w_{n} \rightarrow w$ and $\bar{w} \rightarrow \bar{w}$ as $n \rightarrow \infty$. Further,

$$
\begin{aligned}
d(s, S(x)) & \leq\left\|s-s_{n}\right\|+d\left(s_{n}, S(x)\right) \\
& \leq\left\|s-s_{n}\right\|+D\left(S\left(x_{n}\right), S(x)\right) \\
& \leq\left\|s-s_{n}\right\|+k_{S}\left\|x_{n}-x\right\| \\
& \rightarrow 0 \quad \text { as } n \rightarrow \infty, \\
d(\bar{s}, S(y)) & \leq\left\|\bar{s}-\bar{s}_{n}\right\|+d\left(\bar{s}_{n}, S(y)\right) \\
& \leq\left\|\bar{s}-\bar{s}_{n}\right\|+D\left(S\left(y_{n}\right), S(y)\right) \\
& \leq\left\|\bar{s}-\bar{s}_{n}\right\|+k_{S}\left\|y_{n}-y\right\| \\
& \rightarrow 0 \text { as } n \rightarrow \infty .
\end{aligned}
$$


Similarly, we obtain

$$
\begin{aligned}
d(t, T(x)) & \leq\left\|t-t_{n}\right\|+k_{T}\left\|x_{n}-x\right\| \\
& \rightarrow 0 \quad \text { as } \quad n \rightarrow \infty, \\
d(\bar{t}, T(y)) & \leq\left\|\bar{t}-\bar{t}_{n}\right\|+k_{T}\left\|y_{n}-y\right\| \\
& \rightarrow 0 \quad \text { as } \quad n \rightarrow \infty, \\
d(w, W(x)) & \leq\left\|w-w_{n}\right\|+k_{W}\left\|x_{n}-x\right\| \\
& \rightarrow 0 \quad \text { as } \quad n \rightarrow \infty, \\
d(\bar{w}, W(y)) & \leq\left\|\bar{w}-\bar{w}_{n}\right\|+k_{W}\left\|y_{n}-y\right\| \\
& \rightarrow 0 \quad \text { as } \quad n \rightarrow \infty .
\end{aligned}
$$

Since $S(x), S(y), T(x), T(y), W(x), W(y)$ are closed, we have $s \in S(x), \bar{s} \in S(y)$, $t \in T(x), \bar{t} \in T(y), w \in W(x), \bar{w} \in W(y)$. By the continuities of $f, A, B, N$, $R_{M_{1}(\cdot,), \rho}^{B}, R_{M_{2}(\cdot, \cdot), \lambda}^{B}$ and Algorithm 3.1, we know that $x, y, s, \bar{s}, t, \bar{t}, w, \bar{w}$ satisfy the following relations:

$$
\begin{gathered}
x=R_{M_{1}(\cdot, \cdot), \rho}^{B}\left[\rho a_{1}+B(y)-\rho A(x)+\rho N(s, t, w)\right], \\
y=R_{M_{2}(\cdot, \cdot), \lambda}^{B}\left[\lambda a_{2}+B(x)-\lambda A(x)+\lambda N(\bar{s}, \bar{t}, \bar{w})\right] .
\end{gathered}
$$

By Theorem 3.1, $(x, y, s, \bar{s}, t, \bar{t}, w, \bar{w})$ is a solution of problem (3.1). This completes the proof.

Remark 4.1. (i) If $x=y, \rho=1$ and $\lambda=0$, then Theorem 4.1 reduces to Theorem 4.2 in $[9]$.

(ii) If $x=y, \rho=1, \lambda=0, g=I$ is an identity mapping on $E$ and $M_{1}$ is a general $H$-monotone mapping in the first argument, then Theorem 4.1 reduces to Theorem 3.2 in $[6]$.

(iii) If $x=y, \rho=1, \lambda=0, a_{1}=0, N=0, M_{1}(f(x), g(x))=M_{1}(f(x))$ and $M_{1}$ is a general $H$-monotone mapping, then Theorem 4.1 reduces to Theorem 3.4 in $[11]$.

\section{References}

[1] C. Baiocchi and A. Capelo, Variational and Quasi-Variational Inequalities, J. Wiley and Sons, New York, 1984. 
[2] H. Brezis, Operateur Maximauz Monotone et Semigroupes de Contractions dans les Espaces de Hilbert, North-Holland, Amsterdam, 1973.

[3] R. W. Cottle, F. Giannessi and J. L. Lions, Variational Inequalities: Theory and Applications, J. Wiley and Sons, New York, 1980.

[4] J. Crank, Free and Moving Boundary Problems, Clarendon Press, Oxford, U.K. 1984.

[5] V. F. Demyanov, G. E. Stavroulakis, L. N. Polyakova and P. D. Panagiotopoulos, Quasidifferentiability and Nonsmooth Modelling in Mechanics, Engineering and Economics, Kluwer Academic Publications, Holland, 1996.

[6] X. P. Ding and H. R. Feng, Algorithm for solving a new class of generalized nonlinear implicit quasi-variational inclusions in Banach spaces, Appl. Math. Comput., 208(2009), 547-555.

[7] G. Duvaut and J. L. Lions, Inequalities in Mechanics and Physics, Springer-Verlag, Berlin, 1976.

[8] Y. P. Fang and N. J. Huang, H-monotone operator and resolvent operator technique for variational inclusions, Appl. Math. Comput., 145(2003), 795-803.

[9] Xue-ping Luo and Nan-jing Huang, A new class of variational inclusions with Bmonotone operators in Banach spaces, J. Comput. Appl. Math., 233(2010), 18881896.

[10] S. B. Nadler, Multi-valued contraction mappings, Pacific J. Math., 30(1969), 475-488.

[11] F. Q. Xia and N. J. Huang, Variational inclusions with a general H-monotone operator in Banach spaces, Comput. Math. Appl., 54(2007), 24-30. 